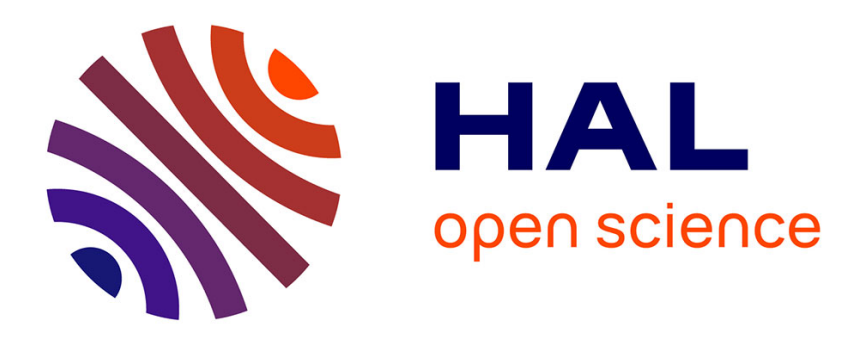

\title{
Ergodic decomposition for measures quasi-invariant under a Borel action of an inductively compact group
}

\author{
Alexander I. Bufetov
}

\section{To cite this version:}

Alexander I. Bufetov. Ergodic decomposition for measures quasi-invariant under a Borel action of an inductively compact group. Sbornik: Mathematics, 2014. hal-01256098

\section{HAL Id: hal-01256098 \\ https://hal.science/hal-01256098}

Submitted on 14 Jan 2016

HAL is a multi-disciplinary open access archive for the deposit and dissemination of scientific research documents, whether they are published or not. The documents may come from teaching and research institutions in France or abroad, or from public or private research centers.
L'archive ouverte pluridisciplinaire HAL, est destinée au dépôt et à la diffusion de documents scientifiques de niveau recherche, publiés ou non, émanant des établissements d'enseignement et de recherche français ou étrangers, des laboratoires publics ou privés.

$$
\text { Copyright }
$$




\title{
ERGODIC DECOMPOSITION FOR MEASURES QUASI-INVARIANT UNDER BOREL ACTIONS OF INDUCTIVELY COMPACT GROUPS
}

\begin{abstract}
ALEXANDER I. BUFETOV
ABSTRACT. The aim of this paper is to prove ergodic decomposition theorems for probability measures quasi-invariant under Borel actions of inductively compact groups (Theorem 11) as well as for $\sigma$-finite invariant measures (Corollary 1 ). For infinite measures the ergodic decomposition is not unique, but the measure class of the decomposing measure on the space of projective measures is uniquely defined by the initial invariant measure (Theorem 2).
\end{abstract}

\section{INTRODUCTION.}

1.1. Outline of the main results. The first result of this paper establishes existence and uniqueness of ergodic decomposition for probability measures quasi-invariant under Borel actions of inductively compact groups (Theorem 1). First we show in Proposition 2 that for actions of inductively compact group ergodicity of a quasi-invariant measure is equivalent to its indecomposability (as Kolmogorov's example [5] shows, this equivalence does not hold for measure-preserving actions of general groups). The ergodic decomposition is then constructed under the additional assumption that the Radon-Nikodym cocycle of the measure is continuous in restriction to each orbit of the group (the fibrewise continuity condition). This condition is only restrictive for actions of uncountable groups. The proof of Theorem 1 relies on Rohlin's method of constructing ergodic decompositions.

Theorem 1 is then applied to $\sigma$-finite invariant measures. In this case the ergodic decomposition is not unique. The measure class of the decomposing measure on the space of projective measures is however uniquely defined by the initial invariant measure (Theorem 2). In the sequel [3] to this paper, its results are applied to the ergodic decomposition of infinite HuaPickrell measures, introduced by Borodin and Olshanski [2], on spaces of infinite Hermitian matrices.

For completeness of the exposition, Kolmogorov's example of a group action admitting decomposable ergodic measures is also included. 
For actions of the group $\mathbb{Z}$ with a quasi-invariant measure, the ergodic decomposition theorem was obtained by Kifer and Pirogov [7] who used the method of Rohlin [11].

For actions of locally compact groups, a general ergodic decomposition theorem is due to Greschonig and Schmidt [6] whose approach is based on Choquet's theorem (see, e.g., [9]). In order to be able to apply Choquet's theorem, Greschonig and Schmidt use Varadarajan's theorem [14] claiming that every Borel action of a locally compact group admits a continuous realization (see Theorem 3 below). It is not clear whether a similar result holds for inductively compact groups (see the question following Theorem 3 ).

For the natural action of the infinite unitary group on the space of infinite Hermitian matrices, ergodic decomposition of invariant probability measures was constructed by Borodin and Olshanski [2]. Borodin and Olshanski [2] rely on Choquet's Theorem, which, however, cannot be used directly since the space of infinite Hermitian matrices is not compact. Borodin and Olshanski embed the space of probability measures on the space of infinite Hermitian matrices into a larger convex compact metrizable set to which Choquet's Theorem can be applied.

Rohlin's approach to the problem of ergodic decomposition requires neither continuity nor compactness, and the results of this paper apply to all Borel actions of inductively compact groups. The martingale convergence theorem is used instead of the ergodic theorem on which Rohlin's argument relies; the idea of using martingale convergence for studying invariant measures for actions of inductively compact groups goes back to Vershik's note [15].

\subsection{Measurable actions of topological groups on Borel spaces.}

1.2.1. Standard Borel spaces. Let $X$ be a set, and let $\mathcal{B}$ be a sigma-algebra on $X$. The pair $(X, \mathcal{B})$ will be called a standard Borel space if there exists a bijection between $X$ and the unit interval which sends $\mathcal{B}$ to the sigmaalgebra of Borel sets. We will continue to call $\mathcal{B}$ the Borel sigma-algebra, and measures defined on $\mathcal{B}$ will be called Borel measures.

Let $\mathfrak{M}(X)$ be the space of Borel probability measures on $X$. A natural $\sigma$-algebra $\mathcal{B}(\mathfrak{M}(X))$ on the space $\mathfrak{M}(X)$ is defined as follows. Let $A \in X$ be a Borel subset, let $\alpha \in \mathbb{R}$, and let

$$
M_{A, \alpha}=\{\nu \in \mathfrak{M}(X): \nu(A)>\alpha\} .
$$

The $\sigma$-algebra $\mathcal{B}(\mathfrak{M}(X))$ is then the smallest $\sigma$-algebra containing all sets $M_{A, \alpha}, A \in \mathfrak{B}(X), \alpha \in \mathbb{R}$. Clearly, if $(X, \mathcal{B})$ is a standard Borel space, then $(\mathfrak{M}(X), \mathcal{B}(\mathfrak{M}(X))$ is also a standard Borel space. 
A Borel measure $\nu$ on a standard $\operatorname{Borel}$ space $(X, \mathcal{B})$ is called $\sigma$-finite if there exists a countable family of disjoint Borel subsets

$$
X_{1}, X_{2}, \ldots, X_{n}, \ldots
$$

of $X$ such that

$$
X=\bigcup_{n=1}^{\infty} X_{n}
$$

and such that $\nu\left(X_{n}\right)<+\infty$ for any $n \in \mathbb{N}$. We denote by $\mathfrak{M}^{\infty}(X)$ the space of all $\sigma$-finite Borel measures on $X$ (note that, in our terminology, finite measures are also $\sigma$-finite). The space $\mathfrak{M}^{\infty}(X)$ admits a natural Borel structure: the Borel $\sigma$-algebra is generated by sets of the form

$$
\left\{\nu \in \mathfrak{M}^{\infty}(X): \alpha<\nu(A)<\beta\right\},
$$

where $\alpha, \beta$ are real and $A$ is a Borel subset of $X$.

If $\nu$ is a Borel measure on $X$ and $f \in L_{1}(X, \nu)$, then for brevity we denote

$$
\nu(f)=\int_{X} f d \nu .
$$

As usual, by a measure class we mean the family of all sigma-finite Borel measures with the same sigma-algebra of sets of measure zero. The measure class of a measure $\nu$ will be denoted $[\nu]$. We write $\nu_{1} \ll \nu_{2}$ if $\nu_{1}$ is absolutely continuous with respect to $\nu_{2}$, while the notation $\nu_{1} \perp \nu_{2}$ means, as usual, that the measures $\nu_{1}, \nu_{2}$ are mutually singular.

1.2.2. Measurable actions of topological groups. Now let $G$ be a topological group endowed with the Borel sigma-algebra. Assume that the group $G$ acts on $X$ and for $g \in G$ let $T_{g}$ be the corresponding transformation. The action will be called measurable (or Borel) if the map

$$
\mathfrak{T}: G \times X \rightarrow X, \quad \mathfrak{T}(g, x)=T_{g} x
$$

is Borel-measurable. The group $G$ acts on $\mathfrak{M}(X)$. It will be convenient for us to consider the right action and for $g \in G$ to introduce the measure

$$
\nu \circ T_{g}(A)=\nu\left(T_{g} A\right) \text {. }
$$

The resulting right action is, of course, Borel.

\subsubsection{Inductively compact groups. Let}

$$
K(1) \subset K(2) \subset \ldots \subset K(n) \subset \ldots
$$

be an ascending chain of metrizable compact groups and set

$$
G=\bigcup_{n=1}^{\infty} K(n) .
$$


The group $G$ will then be called inductively compact. Natural examples are the infinite symmetric group

$$
S(\infty)=\bigcup_{n=1}^{\infty} S(n)
$$

or the infinite unitary group

$$
U(\infty)=\bigcup_{n=1}^{\infty} U(n)
$$

(in both examples, the inductive limit is taken with respect to the natural inclusions).

An inductively compact group $G$ is endowed with the natural topology of the inductive limit, under which a function on $G$ is continuous if and only if it is continuous in restriction to each $K(n)$. The Borel $\sigma$-algebra on $G$ is the span of the Borel $\sigma$-algebras on $K(n), n \in \mathbb{N}$.

\subsection{Cocycles and measures.}

1.3.1. Measurable cocycles. In this paper, a measurable cocycle over a measurable action $\mathfrak{T}$ of a topological group $G$ will always mean a positive real-valued multiplicative cocycle, that is, a measurable map

$$
\rho: G \times X \rightarrow \mathbb{R}_{>0}
$$

satisfying the cocycle identity

$$
\rho(g h, x)=\rho\left(g, T_{h} x\right) \cdot \rho(h, x) .
$$

Given a positive real-valued multiplicative cocycle $\rho$ over a measurable action $\mathfrak{T}$ of a topological group $G$, introduce the space $\mathfrak{M}(\mathfrak{T}, \rho) \subset \mathfrak{M}(X)$ of Borel probability measures with Radon-Nikodym cocycle $\rho$ with respect to the action $\mathfrak{T}$ :

$\mathfrak{M}(\mathfrak{T}, \rho)=\left\{\nu \in \mathfrak{M}(X): \frac{d \nu \circ T_{g}}{d \nu}(x)=\rho(g, x)\right.$ for all $g \in G$ and $\nu$-almost all $\left.x \in X\right\}$.

Note that for a given probability measure $\nu$, quasi-invariant under the action $\mathfrak{T}$, its Radon-Nikodym cocycle is not uniquely, but only almost uniquely defined: if two Radon-Nikodym cocycles $\rho_{1}, \rho_{2}$ corresponding to the same measure $\nu$ are given, then for any $g \in G$ the equality

$$
\rho_{1}(g, x)=\rho_{2}(g, x)
$$

holds for $\nu$-almost all $x \in X$.

Nonetheless, the space $\mathfrak{M}(\mathfrak{T}, \rho)$ is a convex cone. Indeed, if

$$
\nu_{i} \circ T_{g}(A)=\int_{A} \rho(g, x) d \nu_{i}, \quad i=1,2
$$


then also

$$
\left(\nu_{1}+\nu_{2}\right) \circ T_{g}(A)=\int_{A} \rho(g, x) d\left(\nu_{1}+\nu_{2}\right) .
$$

1.3.2. Indecomposability and ergodicity. As before, let $\rho$ be a positive realvalued multiplicative measurable cocycle over a measurable action $\mathfrak{T}$ of a topological group $G$ on a standard Borel space $(X, \mathcal{B})$.

A measure $\nu \in \mathfrak{M}(\mathfrak{T}, \rho)$ is called indecomposable in $\mathfrak{M}(\mathfrak{T}, \rho)$ if the equality $\nu=\alpha \nu_{1}+(1-\alpha) \nu_{2}$, with $\alpha \in(0,1), \nu_{1}, \nu_{2} \in \mathfrak{M}(\mathfrak{T}, \rho)$ implies $\nu=\nu_{1}=\nu_{2}$.

Recall that a Borel set $A$ is called almost invariant with respect to a Borel measure $\nu$ if for every $g \in G$ we have $\nu\left(A \triangle T_{g} A\right)=0$. Indecomposability can be equivalently reformulated as follows.

Proposition 1. A Borel probability measure $\nu \in \mathfrak{M}(\mathfrak{T}, \rho)$ is indecomposable in $\mathfrak{M}(\mathfrak{T}, \rho)$ if and only if any Borel set $A$, almost-invariant under the action $\mathfrak{T}$ with respect to the measure $\nu$, satisfies either $\nu(A)=0$ or $\nu(X \backslash A)=0$.

A measure $\nu \in \mathfrak{M}(\mathfrak{T}, \rho)$ is called ergodic if for every $G$-invariant Borel set $A$ we have either $\nu(A)=0$ or $\nu(X \backslash A)=0$. The set of all ergodic measures with Radon-Nikodym cocycle $\rho$ is denoted $\mathfrak{M}_{\operatorname{erg}}(\mathfrak{T}, \rho)$.

Indecomposable measures are a fortiori ergodic. For actions of general groups, ergodic probability measures may fail to be indecomposable: as Kolmogorov showed, the two notions are different for the natural action of the group of all bijections of $\mathbb{Z}$ on the space of bi-infinite binary sequences (for completeness, we recall Kolmogorov's example in the last Section). An informal reason is that actions of "large" groups may have "too few" orbits (a countable set in Kolmogorov's example), and consequently a convex combination of distinct ergodic measures may also be ergodic.

Nevertheless, for actions of inductively compact groups, the two notions coincide:

Proposition 2. Let $\mathfrak{T}$ be a measurable action of an inductively compact group $G$ on a standard Borel space $(X, \mathcal{B})$, and let $\rho$ be a positive measurable multiplicative cocycle over $\mathfrak{T}$. If a measure $\nu \in \mathfrak{M}(\mathfrak{T}, \rho)$ is ergodic, then $\nu$ is indecomposable in $\mathfrak{M}(\mathfrak{T}, \rho)$.

\subsection{Ergodic decomposition of quasi-invariant probability measures.}

1.4.1. Fibrewise continuous cocycles. To formulate the ergodic decomposition theorem for quasi-invariant measures, we need additional assumptions on the Radon-Nikodym cocycle $\rho$.

Let $\mathfrak{T}$ be a measurable action of a topological group $G$ on a standard Borel space $(X, \mathcal{B})$. 
Definition. A positive real-valued measurable cocycle $\rho: G \times X \rightarrow \mathbb{R}_{>0}$ over the action $\mathfrak{T}$ will be called fibrewise continuous if for any $x \in X$ the function $\rho_{x}: G \rightarrow \mathbb{R}_{>0}$ given by the formula $\rho_{x}(g)=\rho(g, x)$ is continuous.

Remark. If $G$ is inductively compact,

$$
G=\bigcup_{n=1}^{\infty} K(n), \quad K(n) \subset K(n+1)
$$

then, by definition of the inductive limit topology, the requirement of fibrewise continuity precisely means that for any $n \in \mathbb{N}$ the function $\rho_{x}$ defined above is continuous in restriction to $K(n)$.

For general actions of topological groups, it is not clear whether the set of measures with a given Radon-Nikodym cocycle is Borel. That is the case, however, for actions of inductively compact groups and fibrewise continuous cocycles:

Proposition 3. Let $\rho$ be a fibrewise continuous cocycle over a measurable action $\mathfrak{T}$ of a separable metrizable group $G$ on a standard Borel space $(X, \mathcal{B})$. Then the set $\mathfrak{M}(\mathfrak{T}, \rho)$ is a Borel subset of $\mathfrak{M}(X)$.

Indeed, for fixed $g \in G$ the set

$$
\left\{\nu \in \mathfrak{M}(X): \frac{d \nu \circ T_{g}}{d \nu}=\rho(g, x)\right\}
$$

is clearly Borel. Choosing a countable dense subgroup in $G$, we obtain the result.

In Proposition 10 below, we shall see that for a measurable action of an inductively compact group, the set of ergodic measures with a given fibrewise continuous Radon-Nikodym cocycle is Borel as well.

1.4.2. Integrals over the space of measures. Let $\tilde{\nu} \in \mathfrak{M}(\mathfrak{M}(X))$, in other words, let $\tilde{\nu}$ be a Borel probability measure on the space of Borel probability measures on $X$. Introduce a measure $\nu \in \mathfrak{M}(X)$ by the formula

$$
\nu=\int_{\mathfrak{M}(X)} \eta d \tilde{\nu}(\eta)
$$

The integral in the right-hand side of (1) is understood in the following weak sense. For any Borel set $A \subset X$, the function $\operatorname{int}_{A}: \mathfrak{M}(X) \rightarrow \mathbb{R}$ given by the formula $\operatorname{int}_{A}(\eta)=\eta(A)$ is clearly Borel measurable. The equality (1) means that for any Borel set $A \subset X$ we have

$$
\nu(A)=\int_{\mathfrak{M}(X)} \eta(A) d \tilde{\nu}(\eta)
$$




\subsubsection{The ergodic decomposition theorem.}

Theorem 1. Let $\mathfrak{T}$ be a measurable action of an inductively compact group $G$ on a standard Borel space $(X, \mathcal{B})$. Let $\rho$ be a fibrewise continuous positive real-valued multiplicative cocycle over $\mathfrak{T}$. There exists a Borel subset $\widetilde{X} \subset X$ and a surjective Borel map

$$
\pi: \tilde{X} \rightarrow \mathfrak{M}_{\text {erg }}(\rho, \mathfrak{T})
$$

such that

(1) For any $\eta \in \mathfrak{M}_{\text {erg }}(\rho, \mathfrak{T})$ we have $\eta\left(\pi^{-1}(\eta)\right)=1$,

(2) For any $\nu \in \mathfrak{M}(\rho, \mathfrak{T})$ we have

$$
\nu=\int_{\mathfrak{M}_{\text {erg }}(\rho, \mathfrak{T})} \eta d \bar{\nu}(\eta),
$$

where $\bar{\nu}=\pi_{*} \nu$. In particular, for any $\nu \in \mathfrak{M}(\rho, \mathfrak{T})$ we have $\nu(\widetilde{X})=1$.

(3) The correspondence $\nu \rightarrow \bar{\nu}$ is a Borel isomorphism between Borel spaces $\mathfrak{M}(\mathfrak{T}, \rho)$ and $\mathfrak{M}\left(\mathfrak{M}_{\operatorname{erg}}(\mathfrak{T}, \rho)\right)$, and if $\nu \in \mathfrak{M}(\mathfrak{T}, \rho)$ and $\tilde{\nu} \in$ $\mathfrak{M}\left(\mathfrak{M}_{\operatorname{erg}}(\mathfrak{T}, \rho)\right)$ are such that we have

$$
\nu=\int_{\mathfrak{M}_{\operatorname{erg}}(\mathfrak{T}, \rho)} \eta d \tilde{\nu}(\eta),
$$

then $\tilde{\nu}=\bar{\nu}$.

(4) For any $\nu_{1}, \nu_{2} \in \mathfrak{M}_{\operatorname{erg}}(\mathfrak{T}, \rho)$, we have $\nu_{1} \ll \nu_{2}$ if and only if $\bar{\nu}_{1} \ll$ $\bar{\nu}_{2}$, and $\nu_{1} \perp \nu_{2}$ if and only if $\bar{\nu}_{1} \perp \bar{\nu}_{2}$.

\subsection{Ergodic Decomposition of Infinite Invariant Measures.}

1.5.1. Reduction to an equivalent finite measure. We now apply the above results to Borel actions preserving an infinite measure. Given a measurable action $\mathfrak{T}$ of the group $G$, we denote by $\mathfrak{M}_{\text {inv }}^{\infty}(\mathfrak{T})$ the subset of $G$-invariant measures in $\mathfrak{M}^{\infty}$, by $\mathfrak{M}_{\mathrm{erg}}^{\infty}(\mathfrak{T})$ the subset of $G$-invariant ergodic measures in $\mathfrak{M}^{\infty}$. It is not clear whether the sets $\mathfrak{M}_{\text {inv }}^{\infty}(\mathfrak{T})$ and $\mathfrak{M}_{\mathrm{erg}}^{\infty}(\mathfrak{T})$ are Borel. It will be therefore convenient to consider smaller subsets of $\mathfrak{M}^{\infty}$, namely, of measures that assign finite integral to a given positive measurable function.

To simplify notation, consider the space $X$ fixed and omit it from notation, writing, for instance, $\mathfrak{M}$ instead of $\mathfrak{M}(X)$. Also, for a measure $\nu \in \mathfrak{M}^{\infty}$ and $f \in L_{1}(X, \nu)$ write

$$
\nu(f)=\int f d \nu
$$


Given a positive measurable function $f$ on $X$, we set

$$
\mathfrak{M}_{f}^{\infty}=\left\{\nu \in \mathfrak{M}^{\infty}: f \in L_{1}(X, \nu)\right\} .
$$

Introduce a map

$$
P_{f}: \mathfrak{M}_{f}^{\infty} \longrightarrow \mathfrak{M}
$$

by the formula

$$
P_{f}(\nu)=\frac{f \nu}{\nu(f)}
$$

Introduce a cocycle $\rho_{f}$ over the action $\mathfrak{T}$ by the formula

$$
\rho_{f}(g, x)=\frac{f\left(T_{g} x\right)}{f(x)} .
$$

A measure $\nu \in \mathfrak{M}_{f}^{\infty}$ is $\mathfrak{T}$-invariant if and only if

$$
P_{f}(\nu) \in \mathfrak{M}\left(\mathfrak{T}, \rho_{f}\right)
$$

Denote

$$
\begin{gathered}
\mathfrak{M}_{f, 1}^{\infty}=\left\{\nu \in \mathfrak{M}_{f}^{\infty}: \nu(f)=1\right\} \\
\mathfrak{M}_{f, 1, \mathrm{inv}}^{\infty}(\mathfrak{T})=\mathfrak{M}_{f, 1}^{\infty} \cap \mathfrak{M}_{\mathrm{inv}}^{\infty}(\mathfrak{T}) \\
\mathfrak{M}_{f, 1, \mathrm{erg}}^{\infty}(\mathfrak{T})=\mathfrak{M}_{f, 1}^{\infty} \cap \mathfrak{M}_{\mathrm{erg}}^{\infty}(\mathfrak{T})
\end{gathered}
$$

The set $\mathfrak{M}_{f, 1}^{\infty}$ is Borel by definition. The map $P_{f}$ yields a Borel isomorphism of Borel spaces $\mathfrak{M}_{f, 1}^{\infty}$ and $\mathfrak{M}$; the former is consequently a standard Borel space. Furthermore, we clearly have

$$
\begin{aligned}
& P_{f}\left(\mathfrak{M}_{f, 1, \text { inv }}^{\infty}\right)=\mathfrak{M}\left(\mathfrak{T}, \rho_{f}\right) \\
& P_{f}\left(\mathfrak{M}_{f, 1, \mathrm{erg}}^{\infty}\right)=\mathfrak{M}_{\mathrm{erg}}\left(\mathfrak{T}, \rho_{f}\right) .
\end{aligned}
$$

In order to be able to apply Theorem 1 to $\mathfrak{M}\left(\mathfrak{T}, \rho_{f}\right)$, we need an additional assumption on the function $f$.

Definition. A Borel measurable function $f: X \rightarrow \mathbb{R}$ is said to be $f$ brewise continuous if for any $x \in X$ the function $f\left(T_{g} x\right)$ is continuous in $g \in G$.

In particular, if $X$ is a metric space, and the action $\mathfrak{T}$ is itself continuous, then any continuous function is a fortiori fibrewise continuous. To produce continuous integrable functions, one can use the following simple proposition.

Proposition 4. Let $X$ be a metric space, and let $\nu$ be a sigma-finite Borel measure on $X$ assigning finite weight to every ball. Then the space $L_{1}(X, \nu)$ contains a positive continuous function. 
Proof. Let $d$ be the metric on $X$, take $x_{0} \in X$, let $\psi: \mathbb{R}_{+} \rightarrow \mathbb{R}_{>0}$ be positive, bounded and continuous, and set $f(x)=\psi\left(d\left(x, x_{0}\right)\right)$. The mass of every ball is finite, so, if the function $\psi$ decays fast enough at infinity, then $f \in L_{1}(X, \nu)$.

If the function $f$ is fibrewise continuous then the cocycle $\rho_{f}$ given by the formula

$$
\rho_{f}(g, x)=\frac{f\left(T_{g} x\right)}{f(x)}
$$

is fibrewise continuous as well. Consequently, the sets $M_{f, 1 \text {,inv }}^{\infty}$ and $M_{f, 1 \text {,erg }}^{\infty}$ are Borel subsets of $\mathfrak{M}^{\infty}$, and so are the sets $\mathfrak{M}_{f, \text { inv }}^{\infty}$ and $\mathfrak{M}_{f, \text { erg }}^{\infty}$.

Without losing generality assume $\nu(f)=1$ and consider the ergodic decomposition

$$
f \nu=\int_{\mathfrak{M}_{\operatorname{erg}}\left(\mathfrak{T}, \rho_{f}\right)} \eta d \check{\nu}(\eta)
$$

of the measure $f \nu$ in $\mathfrak{M}\left(\mathfrak{T}, \rho_{f}\right)$. Dividing by $f$, we now obtain an ergodic decomposition

$$
\nu=\int_{\mathfrak{M}_{f, 1, e r g}^{\infty}} \eta d \tilde{\nu}(\eta)
$$

of the initial measure $\nu$; note that, by construction, the correspondence $\nu \rightarrow$ $\tilde{\nu}$ is bijective.

Theorem 1 now implies the following

Corollary 1. Let $\mathfrak{T}$ be a measurable action of an inductively compact group $G$ on a standard Borel space $(X, \mathcal{B})$. Let $f: X \rightarrow \mathbb{R}_{>0}$ be measurable, positive and fibrewise continuous. Then:

(1) The sets $\mathfrak{M}_{f, 1, \mathrm{inv}}^{\infty}(\mathfrak{T})$ and $\mathfrak{M}_{f, 1, \mathrm{erg}}^{\infty}(\mathfrak{T})$ are Borel subsets of $\mathfrak{M}^{\infty}(X)$.

(2) Every measure $\eta \in \mathfrak{M}_{f, 1, \mathrm{erg}}^{\infty}(\mathfrak{T})$ is indecomposable in $\mathfrak{M}_{f, 1, \mathrm{inv}}^{\infty}(\mathfrak{T})$.

(3) For any $\nu \in \mathfrak{M}_{f, 1, \text { inv }}^{\infty}(\mathfrak{T})$ there exists a unique Borel probability measure $\bar{\nu}$ on $\mathfrak{M}_{f, 1, \mathrm{erg}}^{\infty}(\mathfrak{T})$ such that

$$
\nu=\int_{\mathfrak{M}_{f, 1, \operatorname{erg}}^{\infty}(\mathfrak{T})} \eta d \bar{\nu}(\eta) .
$$

The bijective correspondence $\nu \rightarrow \bar{\nu}$ is a Borel isomorphism of Borel spaces $\mathfrak{M}_{f, 1, \text { inv }}^{\infty}(\mathfrak{T})$ and $\mathfrak{M}\left(\mathfrak{M}_{f, 1, \mathrm{erg}}^{\infty}(\mathfrak{T})\right)$.

Corollary 1 immediately implies 
Corollary 2. Let $\mathfrak{T}$ be a measurable action of an inductively compact group $G$ on a standard Borel space $(X, \mathcal{B})$, and let $\nu$ be a $\sigma$-finite $\mathfrak{T}$-invariant Borel measure on $X$ such that the space $L_{1}(X, \nu)$ contains a positive Borel measurable fibrewise continuous function. Then the measure $\nu$ admits an ergodic decomposition.

Indeed, an ergodic decomposition is obtained by taking the positive Borel measurable fibrewise continuous function $f \in L_{1}(X, \nu)$, and dividing by $f$ the decomposition (6) of the measure $f \nu$. Such an ergodic decomposition is of course not unique and depends on the choice of the positive Borel measurable fibrewise continuous integrable function.

It is convenient to allow more general ergodic decompositions of infinite measures. Given a measure $\nu \in \mathfrak{M}^{\infty}(X)$ and a $\sigma$-finite Borel measure $\bar{\nu}$ on $\mathfrak{M}^{\infty}(X)$, the equality

$$
\nu=\int_{\mathfrak{M} \infty(X)} \eta d \bar{\nu}(\eta)
$$

will always be understood in a similar way as above, in the following weak sense. Given a Borel set $A$, as above we consider the function

$$
\operatorname{int}_{A}: \mathfrak{M}^{\infty} \rightarrow \mathbb{R}_{\geq 0} \cup\{\infty\}
$$

defined by

$$
\operatorname{int}_{A}(\eta)=\eta(A) .
$$

The equality (7) means that for any Borel set $A$ satisfying $\nu(A)<+\infty$ we have $\operatorname{int}_{A} \in L_{1}\left(\mathfrak{M}^{\infty}(X), \bar{\nu}\right)$ and

$$
\nu(A)=\int_{\mathfrak{M} \infty(X)} \eta(A) d \bar{\nu}(\eta) .
$$

For a measure $\nu$ invariant under the action $\mathfrak{T}$, a decomposition

$$
\nu=\int_{\mathfrak{M}^{\infty}(X)} \eta d \bar{\nu}(\eta)
$$

will be called an ergodic decomposition of $\nu$ if $\bar{\nu}$ is a $\sigma$-finite measure on $\mathfrak{M}^{\infty}(X)$ and $\bar{\nu}$-almost all measures $\eta \in \mathfrak{M}^{\infty}(X)$ are invariant and ergodic with respect to the action $\mathfrak{T}$. Such decomposition is, of course, far from unique: indeed, if

$$
\varphi: \mathfrak{M}^{\infty}(X) \rightarrow \mathbb{R}
$$


is a Borel measurable function such that $\varphi(\eta)>0$ for $\bar{\nu}$-almost all $\eta$, then a new decomposition is obtained by writing

$$
\nu=\int_{\mathfrak{M} \infty(X)} \frac{\eta}{\varphi(\eta)} d(\varphi(\eta) \bar{\nu}(\eta)) .
$$

1.5.2. Projective measures and admissibility. As before, we consider the space $X$ fixed and omit it from notation. Introduce the projective space $\mathbb{P} \mathfrak{M}^{\infty}$, the quotient of $\mathfrak{M}^{\infty}$ by the projective equivalence relation $\sim$ defined in the usual way:

$$
\nu_{1} \sim \nu_{2} \quad \text { if } \quad \nu_{1}=\lambda \nu_{2} \quad \text { for some } \lambda>0 .
$$

Let

$$
\mathbf{p}: \mathfrak{M}^{\infty} \rightarrow \mathbb{P M}^{\infty}
$$

be the natural projection map. Elements of $\mathbb{P M}^{\infty}$ will be called projective measures; finiteness, invariance, quasi-invariance and ergodicity of projective measures are defined in the obvious way, and we denote

$$
\mathbb{P}_{\mathfrak{M}_{\text {inv }}^{\infty}}^{\infty}(\mathfrak{T})=\mathbf{p}\left(\mathfrak{M}_{\text {inv }}^{\infty}(\mathfrak{T})\right) ; \mathbb{P M}_{\text {erg }}^{\infty}(\mathfrak{T})=\mathbf{p}\left(\mathfrak{M}_{\text {erg }}^{\infty}(\mathfrak{T})\right)
$$

The Borel structure in the space $\mathbb{P M}^{\infty}$ is defined in the usual way: a set $A \subset \mathbb{P} \mathfrak{M}^{\infty}$ is Borel if its preimage $\mathbf{p}^{-1}(A)$ is Borel.

Definition. A measure $\bar{\nu} \in \mathfrak{M}^{\infty}\left(\mathfrak{M}^{\infty}\right)$ is called admissible if the projection map $\mathbf{p}$ is $\bar{\nu}$-almost surely a bijection.

For example, any measure supported on the set $\mathfrak{M}^{\infty}(\mathfrak{M})$ or, for a positive measurable $f$, on the set $\mathfrak{M}^{\infty}\left(\mathfrak{M}_{f, 1}^{\infty}\right)$, is automatically admissible.

If the measure $\bar{\nu}$ in an ergodic decomposition (8) is admissible, then the ergodic decomposition is called admissible as well.

The following theorem shows that for a given invariant sigma-finite measure $\nu$, the measure class of the measure $\mathbf{p}_{*} \bar{\nu}$ is the same for all admissible ergodic decompositions (8).

Theorem 2. Let $\mathfrak{T}$ be a measurable action of an inductively compact group $G$ on a standard Borel space $(X, \mathcal{B})$, and let $\nu$ be a $\sigma$-finite $\mathfrak{T}$-invariant Borel measure on $X$ such that the space $L_{1}(X, \nu)$ contains a positive Borel measurable fibrewise continuous function. Then there exists a measure class $P C L(\nu)$ on $\mathbb{P M}^{\infty}$ with the following properties.

(1) For any $\widetilde{\nu} \in P C L(\nu)$ we have $\widetilde{\nu}\left(\mathbb{P} \mathfrak{M}^{\infty} \backslash \mathbb{P M}_{\operatorname{erg}}^{\infty}(\mathfrak{T})\right)=0$.

(2) For any admissible ergodic decomposition

$$
\nu=\int_{\mathfrak{M} \infty} \eta d \bar{\nu}(\eta)
$$

of the measure $\nu$ we have $\mathbf{p}_{*} \bar{\nu} \in P C L(\nu)$. 
(3) Conversely, for any $\sigma$-finite Borel measure $\widetilde{\nu} \in P C L(\nu)$ there exists a unique admissible $\sigma$-finite Borel measure $\bar{\nu}$ on $\mathfrak{M}^{\infty}(X)$ such that $\mathbf{p}_{*} \bar{\nu}=\widetilde{\nu}$ and

$$
\nu=\int_{\mathfrak{M}^{\infty}(X)} \eta d \bar{\nu}(\eta) .
$$

(4) Let $\nu_{1}$ and $\nu_{2}$ be two $\mathfrak{T}$-invariant $\sigma$-finite Borel measures, each admitting a positive fibrewise continuous integrable function. Then $\nu_{1} \ll \nu_{2}$ if and only if $P C L\left(\nu_{1}\right) \ll P C L\left(\nu_{2}\right)$ and $\nu_{1} \perp \nu_{2}$ if and only if $P C L\left(\nu_{1}\right) \perp P C L\left(\nu_{2}\right)$. In particular, $\operatorname{PCL}\left(\nu_{1}\right)=\operatorname{PCL}\left(\nu_{2}\right)$ if and only if $\left[\nu_{1}\right]=\left[\nu_{2}\right]$.

1.5.3. Infinite measures all whose ergodic components are finite. Consider the set $\mathbb{P M}$ of finite projective measures and let $\nu$ be a sigma-finite invariant measure such that $P C L(\nu)$ is supported on $\mathbb{P} \mathfrak{M}$. In this case take an arbitrary ergodic decomposition

$$
\nu=\int_{\mathfrak{M}^{\infty}} \eta d \tilde{\nu}(\eta)
$$

and deform it by writing

$$
\nu=\int_{\mathfrak{M}^{\infty}} \frac{\eta}{\eta(1)} \eta(1) d \tilde{\nu}(\eta) .
$$

In this way we obtain an ergodic decomposition

$$
\nu=\int_{\mathfrak{M}_{\text {erg }}(\mathfrak{T})} \eta d \bar{\nu}(\eta),
$$

where the measure $\bar{\nu}$, supported on $\mathfrak{M}_{\text {erg }}(\mathfrak{T})$, is uniquely defined by $\nu$.

Acknowledgements. Grigori Olshanski posed the problem to me; I am deeply grateful to him. I am deeply grateful to Klaus Schmidt for kindly explaining to me the construction of Section 5 in [6] and for many very helpful discussions. I am deeply grateful to Yves Coudène for kind explanations of Souslin theory. I am deeply grateful to Vadim Kaimanovich and Sevak Mkrtchyan for useful discussions. I am deeply grateful to Nikita Kozin for typesetting parts of the manuscript. Part of this work was done while I was visiting the Institut Mittag-Leffler in Djursholm, the Erwin Schroedinger Institute in Vienna and the Max Planck Institute in Bonn; I am deeply grateful to these institutions for their hospitality.

This work was supported in part by an Alfred P. Sloan Research Fellowship, by the Dynasty Foundation Fellowship, by Grants MK-4893.2010.1 and MK-6734.2012.1 of the President of the Russian Federation, by the Programme on Dynamical Systems and Mathematical Control Theory of 
the Presidium of the Russian Academy of Sciences, by the RFBR-CNRS grant 10-01-93115, by the RFBR grant 11-01-00654 and by the Edgar Odell Lovett Fund at Rice University.

\section{AVERAGING OPERATORS.}

2.1. Averaging over orbits of compact groups. Let $K$ be a compact group endowed with the Haar measure $\mu_{K}$ and let $\mathfrak{T}_{K}$ be a measurable action of $K$ on a standard Borel space $(X, \mathcal{B})$. Let $\rho$ be a positive multiplicative realvalued measurable cocycle over the action $\mathfrak{T}_{K}$. Let $\mathbb{B}(X)$ be the space of bounded measurable functions on $X$ endowed with the Tchebychev metric. Introduce an operator $\mathcal{A}_{K}^{\rho}: \mathbb{B}(X) \rightarrow \mathbb{B}(X)$ by the formula

$$
\left(\mathcal{A}_{K}^{\rho} f\right)(x)= \begin{cases}\frac{\int_{K} f\left(T_{k} x\right) \rho(k, x) d \mu_{K}(k)}{\int_{K} \rho(k, x) d \mu_{K}(k)} & \text { if } \int_{K} \rho(k, x) d \mu_{K}(k)<+\infty \\ 0, & \text { if } \int_{K} \rho(k, x) d \mu_{K}(k)=+\infty .\end{cases}
$$

It is clear that $\mathcal{A}_{K}^{\rho}$ is a positive contraction on the space $\mathbb{B}(X)$.

Let $\mathcal{J}_{K}$ be the $\sigma$-algebra of $K$-invariant subsets of $X$, and, for a given measure $\nu$, let $\mathcal{J}_{K}^{\nu}$ be the completion of $\mathcal{J}_{K}$ with respect to $\nu$.

As before, $\mathfrak{M}\left(\mathfrak{T}_{K}, \rho\right)$ stands for the space of Borel probability measures on $X$ with Radon-Nikodym cocycle $\rho$ with respect to the action $\mathfrak{T}_{K}$.

Lemma 1. For any $\nu \in \mathfrak{M}\left(\mathfrak{T}_{K}, \rho\right)$ and any $f \in L_{1}(X, \nu)$ both integrals on the right-hand side of (9) are $\nu$-almost surely finite. The extended operator $\mathcal{A}_{K}^{\rho}$ is a positive contraction of $L_{1}(X, \nu)$, and we have the $\nu$-almost sure equality

$$
\mathcal{A}_{K}^{\rho} f=\mathbb{E}\left(f \mid \mathcal{J}_{K}^{\nu}\right) .
$$

Remark. Note that the left-hand side of (10) does not depend on the measure $\nu$, only on the cocycle $\rho$. This simple observation will be important in what follows.

Proof. Let $\rho_{x}: K \rightarrow \mathbb{R}$ be defined by the formula

$$
\rho_{x}(k)=\rho(k, x) .
$$

From the Fubini Theorem it immediately follows that for $\nu$-almost every $x \in X$ we have $\rho_{x} \in L_{1}\left(K, \mu_{K}\right)$. Now take $\varphi \in L_{1}(X, \nu)$ and set

$$
\varphi_{x}(k)=\varphi\left(T_{k} x\right) \rho(k, x) .
$$


Proposition 5. For $\nu$-almost every $x \in X$ we have $\varphi_{x} \in L_{1}\left(K, \mu_{K}\right)$.

Consider the product space $K \times X$ endowed with the measure $\tilde{\nu}$ defined by the formula

$$
d \tilde{\nu}=\rho(k, x) d \mu_{K} d \nu
$$

For any fixed $k_{0} \in K$ we have

$$
\int_{X} \rho\left(k_{0}, x\right) d \nu(x)=1
$$

whence $\tilde{\nu}$ is a probability measure.

For any $k \in K$ we have

$$
\int\left|\varphi\left(T_{k} x\right)\right| \cdot \rho(k, x) d \nu(x)=\int|\varphi(x)| d \nu(x),
$$

so the function $\tilde{\varphi}(k, x)=\varphi\left(T_{k} x\right)$ satisfies $\tilde{\varphi} \in L_{1}(K \times X, \tilde{\nu})$. The claim of the Proposition follows now from the Fubini Theorem.

We return to the proof of Lemma 1 . First, the cocycle property implies that

$$
\mathcal{A}_{K}^{\rho} \varphi(x)=\mathcal{A}_{K}^{\rho} \varphi\left(T_{k} x\right)
$$

for any $k \in K$. By the Fubini Theorem applied to the space $K \times X$ endowed with the measure $\tilde{\nu}$, for any Borel subset $A \subset X$ and any $\tilde{\varphi} \in L_{1}(K \times X, \tilde{\nu})$ we have:

$$
\begin{aligned}
& \int_{A} \int_{K} \tilde{\varphi}(k, x) \rho(k, x) d \mu_{K}(k) d \nu(x)= \\
& \quad=\int_{A} \int_{K}\left(\frac{\int_{K} \tilde{\varphi}(k, x) \rho(k, x) d \mu_{K}(k)}{\int_{K} \rho(k, x) d \mu_{K}(k)}\right) \rho(k, x) d \mu_{K}(k) d \nu(x) .
\end{aligned}
$$

Now take $\varphi \in L_{1}(X, \nu)$ and apply the above formula to the function

$$
\tilde{\varphi}(k, x)=\varphi\left(T_{k} x\right)
$$

(note here that $\tilde{\varphi} \in L_{1}(K \times X, \tilde{\nu})$ by Fubini's theorem). We obtain

$$
\int_{K}\left(\int_{A} \varphi\left(T_{k} x\right) d \nu \circ T_{k}(x)\right) d \mu_{K}(k)=\int_{K}\left(\int_{A} \mathcal{A}_{K}^{\rho} \varphi(x) d \nu \circ T_{k}(x)\right) d \mu_{K}(k) .
$$


Now let the set $A$ be $K$-invariant. Recalling that the function $\mathcal{A}_{K}^{\rho} \varphi$ is $K$-invariant as well, we finally obtain

$$
\int_{A} \varphi(x) d \nu(x)=\int_{A} \mathcal{A}_{K}^{\rho} \varphi(x) d \nu(x),
$$

and the Lemma is proved completely.

2.2. Averaging over orbits of inductively compact groups. As above, let

$$
G=\bigcup_{n=1}^{+\infty} K(n), K(n) \subset K(n+1)
$$

be an inductively compact group, and let $\mu_{K(n)}$ denote the Haar measure on the group $K(n)$. Assume we are given a measurable action $\mathfrak{T}$ of $G$ on a standard Borel space $(X, \mathcal{B})$. Let $\mathcal{J}_{K(n)}$ stand for the $\sigma$-algebra of $K(n)-$ invariant measurable subsets of X, and let $\mathcal{J}_{G}$ be the $\sigma$-algebra of $G$-invariant subsets of $X$. Clearly, we have

$$
\mathcal{J}_{G}=\bigcap_{n=1}^{\infty} \mathcal{J}_{K(n)} .
$$

Let $\rho$ be a positive measurable multiplicative cocycle over the action $\mathfrak{T}$.

The averaging operators $\mathcal{A}_{K(n)}^{\rho}, n \in \mathbb{N}$, are defined, for a bounded measurable function $\varphi$ on $X$, by formula (9). For brevity, we shall sometimes write $\mathcal{A}_{n}^{\rho}=\mathcal{A}_{K(n)}^{\rho}$.

Now take $\nu \in \mathfrak{M}(\mathfrak{T}, \rho)$ and let $\mathcal{J}_{K(n)}^{\nu}, \mathcal{J}_{G}^{\nu}$ be the completions of the sigmaalgebras $\mathcal{J}_{K(n)}, \mathcal{J}_{G}$ with respect to the measure $\nu$.

By the results of the previous subsection, for any $\varphi \in L_{1}(X, \nu)$, we have the $\nu$-almost sure equality

$$
\mathcal{A}_{n}^{\rho} \varphi=\mathbb{E}\left(\varphi \mid \mathcal{J}_{K(n)}^{\nu}\right) .
$$

Since $\mathcal{J}_{K(n+1)}^{\nu} \subset \mathcal{J}_{K(n)}^{\nu}$, the reverse martingale convergence theorem implies the following

Proposition 6. For any $\varphi \in L_{1}(X, \nu)$ we have

$$
\lim _{n \rightarrow \infty} \mathcal{A}_{n}^{\rho} \varphi=\mathbb{E}\left(\varphi \mid \mathcal{J}_{G}^{\nu}\right)
$$

both $\nu$-almost surely and in $L_{1}(X, \nu)$.

Introduce the averaging operator $\mathcal{A}_{\infty}^{\rho}$ by setting

$$
\mathcal{A}_{\infty}^{\rho} \varphi(x)=\lim _{n \rightarrow \infty} \mathcal{A}_{n}^{\rho} \varphi(x) .
$$

If for a given $x \in X$ the sequence $\mathcal{A}_{n}^{\rho} \varphi(x)$ fails to converge, then the value $\mathcal{A}_{\infty}^{\rho} \varphi(x)$ is not defined. From the definitions and the Reverse Martingale Convergence Theorem we immediately have 
Proposition 7. A measure $\eta \in \mathfrak{M}(\mathfrak{T}, \rho)$ is ergodic of and only if for any $\varphi \in L_{1}(X, \eta)$ we have

$$
\mathcal{A}_{\infty}^{\rho} \varphi(x)=\int_{X} \varphi d \eta
$$

almost everywhere with respect to the measure $\eta$.

Conversely, we have

Proposition 8. Let $\eta \in \mathfrak{M}(\mathfrak{T}, \rho)$ and assume that there exists a dense set $\Psi \subset L_{1}(X, \eta)$ such that for any $\psi \in \Psi$ we have

$$
\mathcal{A}_{\infty}^{\rho} \psi=\int \psi d \eta
$$

almost surely with respect to $\eta$. Then the measure $\eta$ is ergodic.

\subsection{Equivalence of indecomposability and ergodicity: proof of Propo- sition 2 .}

Proposition 9. Let $A$ be a $G$-almost-invariant Borel subset of $X$. Then there exists a $G$-invariant Borel set $\tilde{A}$ such that

$$
\nu(A \triangle \tilde{A})=0 .
$$

Proof. Let $\chi_{A}$ be, as usual, the indicator function of $A$. If $A$ is $G$-almostinvariant, then for almost every $x \in A$ and all $n \in \mathbb{N}$ we have

$$
\mathcal{A}_{n}^{\rho} \chi_{A}(x)=1 .
$$

Indeed, consider the set $K(n) \times A$ endowed with the product measure $\mu_{K(n)} \times \nu$. For almost all points $(k, x) \in K(n) \times A$ by definition we have $T_{k} x \in A$. By Fubini's theorem, for almost every $x \in X$ the set $\left\{k \in K(n): T_{k} x \in A\right\}$ has full measure, whence $\mathcal{A}_{n}^{\rho} \chi_{A}(x)=1$ as desired.

Now introduce the set $\tilde{A}$ as follows:

$$
\tilde{A}=\left\{x \in X: \mathcal{A}_{n}^{\rho} \chi_{A}(x)=1 \text { for all sufficiently large } n \in \mathbb{N}\right\} .
$$

By definition, $\tilde{A} \supset A$. On the other hand, since for $x \in \tilde{A}$ we have $\mathcal{A}_{\infty}^{\rho} \chi_{A}(x)=1$, the equality

$$
\int_{X} \mathcal{A}_{\infty}^{\rho} \chi_{A} d \nu=\nu(A)
$$

implies $\nu(\tilde{A}) \leq \nu(A)$, whence $\nu(\tilde{A} \triangle A)=0$ and the proposition is proved. 


\subsection{The set of ergodic measures is Borel.}

Proposition 10. Let $\rho$ be a fibrewise continuous cocycle over a measurable action $\mathfrak{T}$ of an inductively compact group $G$ on a standard Borel space $(X, \mathcal{B})$. Then the set $\mathfrak{M}_{\operatorname{erg}}(\mathfrak{T}, \rho)$ is a Borel subset of $\mathfrak{M}(X)$.

Proof. We start with the following auxiliary proposition.

Proposition 11. Let $(X, \mathcal{B})$ be a standard Borel space. There exists a countable set $\Phi$ of bounded measurable functions on $X$ such that for any probability measure $\nu$ on $X$ and any bounded measurable function $\varphi: X \rightarrow \mathbb{R}$ there exists a sequence $\varphi_{n} \in \Phi$ such that

(1) $\sup _{n \in \mathbb{N}, x \in X} \varphi_{n}(x)<+\infty$;

(2) $\varphi \rightarrow \varphi$ as $n \rightarrow \infty$ almost surely with respect to $\nu$.

Proof. On the unit interval take the family of piecewise-linear functions with nodes at rational points.

We return to the proof of Proposition 10. It is clear that for any fixed bounded measurable function $\varphi$ on $X$ the set

$$
\left\{\nu: \lim _{n \rightarrow \infty} \mathcal{A}_{n}^{\rho} \varphi \text { exists and is constant } \nu \text {-almost surely }\right\}
$$

is Borel. Intersecting over all $\varphi \in \Phi$ and using Proposition 8 , we obtain the claim.

\section{THE SIGMA-ALGEBRA OF $G$-INVARIANT SETS.}

\subsection{Measurable partitions in the sense of Rohlin.}

3.1.1. Lebesgue spaces. A triple $(X, \mathcal{B}, \nu)$, where $X$ is a set, $\mathcal{B}$ a sigmaalgebra on $X$, and $\nu$ a measure on $X$, defined on $\mathcal{B}$ and such that $\mathcal{B}$ is complete with respect to $\nu$ is called a Lebesgue space if it is either countable or measurably isomorphic to the unit interval endowed with the sigma-algebra of Lebesgue measurable sets and the Lebesgue measure (perhaps with a countable family of atoms). No Borel structure on $X$ is assumed in this definition.

3.1.2. Measurable partitions. A partition $\xi$ of $X$ is simply a representation of $X$ as a disjoint union of measurable sets:

$$
X=\bigcup X_{\alpha}
$$

The sets $X_{\alpha}$ are called elements of the partition. For a point $x$, the element of the partition $\xi$ containing $x$ will be denoted $\mathcal{C}_{\xi}(x)$. A family of sets $\Psi$ is said to be a basis for the partition $\xi$ if for any two elements $X_{1}, X_{2}$ of $\xi$ there exists a set $A_{1}$ in $\Psi$ containing $A_{1}$ and disjoint from $A_{2}$. A measurable 
partition $\xi$ of $(X, \mathcal{B}, \nu)$ is by definition a partition of a subset $Y \subset X$ of full measure which admits a countable basis.

Following Rohlin, to a measurable partition $\xi$ we assign the quotient space $\bar{X}(\xi)$ whose points are elements of the partition $\xi$. We have a natural almost surely defined projection map $\pi_{\xi}: X \rightarrow \bar{X}(\xi)$, which endows the set $\bar{X}(\xi)$ with a natural sigma-algebra $\overline{\mathcal{B}}(\xi)$, the push-forward of $\mathcal{B}$, and the natural quotient-measure $\bar{\nu}_{\xi}$, the push-forward of the measure $\nu$. Rohlin proved that the space $\left(\bar{X}(\xi), \overline{\mathcal{B}}(\xi), \bar{\nu}_{\xi}\right)$ is again a Lebesgue space. Furthermore, Rohlin showed that the measure $\nu$ admits the canonical system of conditional measures defined as follows. For $\bar{\nu}_{\xi}$-almost every element $\mathcal{C}$ of the partition $\xi$ there is a probability measure $\nu_{\mathrm{e}}$ on $\mathcal{C}$ such that for any set $A \in \mathcal{B}$ the function $\operatorname{int}_{A}: \bar{X}(\xi) \rightarrow \mathbb{R}$ given by the formula $\operatorname{int}_{A}(\mathrm{C})=\nu_{\mathfrak{C}}(A)$ is $\overline{\mathcal{B}}$-measurable and we have

$$
\nu(A)=\int_{\bar{X}(\xi)} \nu_{\mathfrak{C}}(A) d \bar{\nu}_{\xi}(\mathfrak{C}) .
$$

This system of canonical conditional measures is unique: any two systems coincide $\bar{\nu}_{\xi}$-almost surely. To a measurable partition $\xi$ we now assign an averaging operator $\mathcal{A}_{\xi}$ on $L_{1}(X, \nu)$, given by the formula

$$
\mathcal{A}_{\xi} f(x)=\int_{\mathfrak{C}_{\xi}(x)} f(x) d \nu_{\mathrm{C}_{\xi}(x)}
$$

(the right-hand side is defined $\nu$-almost surely by Rohlin's Theorem). Given a measurable partition $\xi$, let $\mathcal{B}_{\xi}$ be the sigma-algebra of measurable subsets of $X$ which are unions of elements of $\xi$ and a set of measure zero. Rohlin proved that for any $f \in L_{1}(X, \nu)$ we have the $\nu$-almost sure identity

$$
\mathbb{E}\left(f \mid \mathcal{B}_{\xi}\right)=\mathcal{A}_{\xi} f
$$

Rohlin showed, furthermore, that every complete sub-sigma-algebra $\mathcal{B}_{1} \subset$ $\mathcal{B}$ has the form $\mathcal{B}_{1}=\mathcal{B}_{\xi}$ for some measurable partition $\xi$ of the Lebesgue space $(X, \mathcal{B}, \nu)$.

3.2. Borel partitions. Let $(X, \mathcal{B})$ be a standard Borel space. A decomposition

$$
X=\bigsqcup_{\alpha} X_{\alpha}
$$

where $\alpha$ takes values in an arbitrary index set and where, for each $\alpha$, the set $X_{\alpha}$ is Borel, will be called a Borel partition if there exists a countable family

$$
Z_{1}, \ldots, Z_{n}, \ldots
$$


of Borel sets such that for any two indices $\alpha_{1}, \alpha_{2}$ where $\alpha_{1} \neq \alpha_{2}$, there exists $i \in \mathbb{N}$ satisfying

$$
X_{\alpha_{1}} \subset Z_{i}, \quad X_{\alpha_{2}} \cap Z_{i}=\emptyset .
$$

In this case, the countable family will be called the countable basis for the partition.

If $\nu$ is a Borel probability measure on $X$, then the space $(X, \mathcal{B}, \nu)$ is a Lebesgue space in the sense of Rohlin, while a Borel partition now becomes a measurable partition in the sense of Rohlin. Observe that all conditional measures are in this case defined on the Borel sigma-algebra.

\subsection{The measurable partition corresponding to the sigma-algebra of} invariant sets. Our first aim is to give an explicit description of the measurable partition corresponding to the $\sigma$-algebra $\mathcal{J}_{G}$ of $G$-invariant sets.

Let $\Phi$ be the set given by Proposition 11 and write $\Phi=\left\{\varphi_{1}, \varphi_{2}, \ldots, \varphi_{n}, \ldots\right\}$.

Introduce a set $X(\Phi, \rho)$ by the formula:

$$
X(\Phi, \rho)=\left\{x \in X: A_{\infty}^{\rho} \varphi_{k}(x) \text { is defined for all } k \in \mathbb{N}\right\} .
$$

The set $X(\Phi, \rho)$ is clearly Borel. Observe that for any $\nu \in \mathfrak{M}(\mathfrak{T}, \rho)$ we have

$$
\nu(\mathfrak{M}(\mathfrak{T}, \rho))=1 .
$$

Let $\mathbb{R}^{\mathbb{N}}$ be the space of all real sequences:

$$
\mathbb{R}^{\mathbb{N}}=\left\{\mathbf{r}=\left(r_{k}\right), k \in \mathbb{N}, r_{k} \in \mathbb{R}\right\} .
$$

We endow $\mathbb{R}^{\mathbb{N}}$ with the usual product $\sigma$-algebra, which turns it into a standard Borel space. For $\mathbf{r} \in \mathbb{R}^{\mathbb{N}}$, we introduce a subset $X(\mathbf{r}, \Phi, \rho)$ by the formula

$$
X(\mathbf{r}, \Phi, \rho)=\left\{x \in X(\Phi, \rho): A_{\infty}^{\rho} \varphi_{k}(x)=r_{k}, k \in \mathbb{N}\right\} .
$$

For any $\mathbf{r} \in \mathbb{R}^{\mathbb{N}}$, the set $X(\mathbf{r}, \Phi, \rho)$ is Borel, and we clearly have

$$
X(\Phi, \rho)=\bigsqcup_{\mathbf{r} \in \mathbb{R}^{\mathbb{N}}} X(\mathbf{r}, \Phi, \rho) .
$$

It is clear from the definitions that the Borel partition

$$
X=(X \backslash X(\Phi, \rho)) \bigsqcup \bigsqcup_{\mathbf{r} \in \mathbb{R}^{\mathbb{N}}} X(\mathbf{r}, \Phi, \rho)
$$

has a countable basis.

Introduce a map

$$
\Pi_{\Phi}: X(\Phi, \rho) \longrightarrow \mathbb{R}^{\mathbb{N}}
$$

by the formula

$$
\Pi_{\Phi}(x)=\left(\mathcal{A}_{\infty}^{\rho} \varphi_{1}(x), \ldots, \mathcal{A}_{\infty}^{\rho} \varphi_{n}(x), \ldots\right) .
$$


The map $\Pi_{\Phi}$ is, by definition, Borel. Now, introduce a map

$$
\operatorname{Int}_{\Phi}: \mathfrak{M}(\mathfrak{T}, \rho) \longrightarrow \mathbb{R}^{\mathbb{N}}
$$

by the formula

$$
\operatorname{Int}_{\Phi}(\nu)=\left(\int_{X} \varphi_{1} d \nu, \ldots, \int_{X} \varphi_{n} d \nu, \ldots\right) .
$$

The map $\operatorname{Int}_{\Phi}$ is, by definition, Borel and injective.

By Souslin's Theorem (see [13], [1], [4]), it follows the sets $\operatorname{Int}_{\Phi}(\mathfrak{M}(\mathfrak{T}, \rho))$ and $\operatorname{Int}_{\Phi}\left(\mathfrak{M}_{\text {erg }}(\mathfrak{T}, \rho)\right)$ are Borel. Introduce a subset $X_{\text {erg }} \subset X$ by the formula

$$
X_{\text {erg }}=\Pi_{\Phi}^{-1}\left(\operatorname{Int}_{\Phi}\left(\mathfrak{M}_{\operatorname{erg}}(\mathfrak{T}, \rho)\right)\right) .
$$

Again, Souslin's Theorem implies that the set $X_{\text {erg }}$ is Borel. We thus have the following diagram, all whose arrows correspond to Borel maps

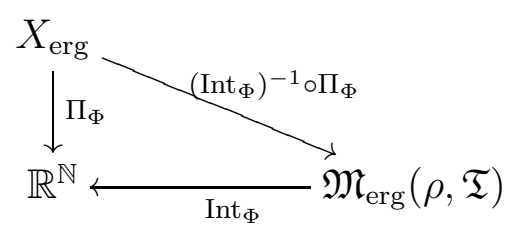

We shall now see that for any $\nu \in \mathfrak{M}(\mathfrak{T}, \rho)$ we have

$$
\nu\left(X_{\text {erg }}\right)=1
$$

Indeed, take an arbitrary $\nu \in \mathfrak{M}(\mathfrak{T}, \rho)$. The Borel partition $\xi$ now induces a measurable partition that we denote $\xi^{\nu}$. Let $\bar{X}\left(\xi^{\nu}\right)$ be the space of elements of the partition $\xi$, or, in other words, the quotient of the space $X$ by the partition $\xi$. Let

$$
\pi_{\xi^{\nu}}: X \longrightarrow \bar{X}\left(\xi^{\nu}\right)
$$

be the natural projection map, and let

$$
\widetilde{\nu}=\left(\pi_{\xi^{\nu}}\right)_{*} \nu
$$

be the quotient measure on $\bar{X}\left(\xi^{\nu}\right)$.

By Rohlin's Theorem, $\widetilde{\nu}$-almost every element $\mathcal{C}$ of the partition $\xi^{\nu}$ carries a canonical conditional measure $\nu_{\mathcal{C}}$. The key step in the construction of the ergodic decomposition is given by the following Proposition.

Proposition 12. The measurable partition $\xi^{\nu}$ generates the $\sigma$-algebra $\mathcal{J}_{G}^{\nu}$, the $\nu$-completion of the $\sigma$-algebra of Borel $G$-invariant sets. For $\widetilde{\nu}$-almost every $\mathcal{C}$ we have $\nu_{\mathrm{e}} \in \mathfrak{M}_{\operatorname{erg}}(\rho, \mathfrak{T})$. 
The Proposition will be proved in the following subsection. Rohlin's decomposition

$$
\nu=\int_{\bar{X}\left(\xi^{\nu}\right)} \nu_{\mathcal{C}} d \widetilde{\nu}(\mathcal{C})
$$

will now be used to obatain an ergodic decomposition of the measure $\nu$.

Indeed, let the map

$$
\operatorname{mes}_{\xi^{\nu}}: \bar{X}\left(\xi^{\nu}\right) \longrightarrow \mathfrak{M}_{\operatorname{erg}}(\rho, \mathfrak{T})
$$

be given by the formula

$$
\operatorname{mes}_{\xi^{\nu}}(\mathrm{C})=\nu_{\mathrm{e}}
$$

Proposition 13. The map $\operatorname{mes}_{\xi^{\nu}}$ is $\widetilde{\nu}$-measurable.

Proof. Let $\varphi$ be a bounded measurable function on $X$. Let $\alpha \in \mathbb{R}$. By definition of the measurable structure on the quotient space $\bar{X}\left(\xi^{\nu}\right)$, it suffices to show that the set

$$
\left\{x \in X: \int \varphi d \nu_{e}(x)>\alpha\right\}
$$

is $\nu$-measurable. But by Proposition 12 we have the $\nu$-almost sure equality

$$
\left\{x \in X: \int \varphi d \nu_{\mathfrak{C}(x)}>\alpha\right\}=\left\{x \in X: \mathcal{A}_{\infty}^{\rho} \varphi(x)>\alpha\right\} .
$$

Since the set $\left\{x \in X: \mathcal{A}_{\infty}^{\rho} \varphi(x)>\alpha\right\}$ is Borel, the Proposition is proved.

For $x \in X$ let $\mathcal{C}_{\xi}(x)$ be the element of the partition $\xi$ containing $x$, and introduce a map

by the formula

$$
\operatorname{Mes}_{\xi^{\nu}}: X \longrightarrow \mathfrak{M}_{\operatorname{erg}}(\rho, \mathfrak{T})
$$

We have a commutative diagram

$$
\operatorname{Mes}_{\xi^{\nu}}(x)=\nu_{\mathrm{e}_{\xi}(x)} .
$$



In particular, the map $\operatorname{Mes}_{\xi^{\nu}}$ is $\nu$-measurable. Proposition 12 immediately implies the following

Corollary 3. For any $\nu \in M_{\mathrm{erg}}(\mathfrak{T}, \rho)$ we have $\nu\left(X_{\mathrm{erg}}\right)=1$. The equality

$$
\operatorname{Mes}_{\xi^{\nu}}=\left(\operatorname{Int}_{\Phi}\right)^{-1} \circ \Pi_{\Phi}
$$

holds $\nu$-almost surely. 


\section{Denoting}

$$
\bar{\nu}=\left(\operatorname{Mes}_{\xi^{\nu}}\right)_{*} \nu=\left(\operatorname{mes}_{\xi^{\nu}}\right)_{*} \widetilde{\nu}
$$

we finally obtain an ergodic decomposition

$$
\nu=\int_{\mathfrak{M}_{\operatorname{erg}}(\rho, \mathfrak{T})} \eta d \bar{\nu}(\eta)
$$

for the measure $\nu$. To complete the proof of the first two claims of Theorem 1 it remains to establish Proposition 12.

\subsection{Proof of Proposition 12.}

\subsubsection{Proof of the first claim.}

Proof. On one hand, every element of the partition $\xi^{\nu}$ is by definition $G$ invariant.

Conversely, let $A$ be $G$-invariant. Our aim is to find a measurable set $A^{\prime}$ which is a union of elements of the partition $\xi^{\nu}$ and satisfies

$$
\nu\left(A \triangle A^{\prime}\right)=0 \text {. }
$$

Take a sequence $\varphi_{n_{k}} \in \Phi$ such that

$$
\sup _{k \in \mathbb{N}, x \in X} \varphi_{n_{k}}(x)<+\infty
$$

and $\varphi_{n_{k}} \rightarrow \chi_{A}$ almost surely with respect to the measure $\nu$ as $k \rightarrow \infty$.

Now let

and let

$$
R_{A}=\left\{\mathbf{r} \in \mathbb{R}^{\mathbb{N}}, \mathbf{r}=\left(r_{n}\right), \lim _{k \rightarrow \infty} r_{n_{k}}=1\right\}
$$

$$
\begin{gathered}
A^{\prime}=\bigcup_{\mathbf{r} \in R_{A}} X(\rho, \Phi, \mathbf{r}), \\
A^{\prime \prime}=\left\{x \in X: \mathcal{A}_{\infty}^{\rho} \chi_{A}(x)=1\right\} .
\end{gathered}
$$

Since $A$ is $G$-invariant, we have

$$
\nu\left(A \triangle A^{\prime \prime}\right)=0 .
$$

Since

$$
\lim _{k \rightarrow \infty} \varphi_{n_{k}}=\chi_{A}
$$

$\nu$-almost surely and all functions are uniformly bounded, we have

$$
\mathcal{A}_{\infty}^{\rho} \varphi_{n_{k}} \rightarrow \mathcal{A}_{\infty}^{\rho} \chi_{A}
$$

almost surely as $k \rightarrow \infty$. It follows that

$$
\nu\left(A^{\prime} \triangle A^{\prime \prime}\right)=0,
$$

and, finally, we obtain

$$
\nu\left(A \triangle A^{\prime}\right)=0,
$$


which is what we had to prove.

\subsubsection{Proof of the second claim.}

Proposition 14. For every $g \in G$, for $\bar{\nu}$-almost every $\mathcal{C} \in \bar{X}\left(\xi^{\nu}\right)$ and $\nu_{\mathrm{e}}$ almost every $x \in X$ we have

$$
\frac{d \nu_{\mathrm{e}} \circ T_{g}}{d \nu_{\mathrm{e}}}(x)=\rho(g, x)
$$

Proof. This is immediate from the uniqueness of the canonical system of conditional measures. Indeed, on the one hand, we have

$$
\nu \circ T_{g}=\int_{\bar{X}\left(\xi^{\nu}\right)} \nu_{\mathrm{e}} \circ T_{g} d \bar{\nu}(\mathrm{C}) ;
$$

on the other hand,

$$
\nu \circ T_{g}=\rho(g, x) \cdot \nu=\int_{\bar{X}\left(\xi^{\nu}\right)} \rho(g, x) \cdot \nu_{\mathcal{C}} d \bar{\nu}(\mathcal{C}),
$$

whence $\nu_{\mathrm{C}} \cdot T_{g}=\rho(g, x) \cdot \nu_{\mathrm{C}}$ for $\widetilde{\nu}$-almost all $\mathrm{C} \in \bar{X}\left(\xi^{\nu}\right)$, and the Proposition is proved.

Fibrewise continuity of the cocycle is necessary to pass from a countable dense subgroup to the whole group.

Proposition 15. Let $\rho$ be a positive Borel fibrewise continuous cocycle over a measurable action $\mathfrak{T}_{K}$ of a compact group $K$ on a standard Borel space $(X, \mathcal{B})$. Let $\nu$ be a Borel probability measure on $X$. Let $K^{\prime} \subset K$ be dense, and assume that the equality

$$
\frac{d \nu \circ T_{k}}{d \nu}=\rho(k, x)
$$

holds for all $k \in K^{\prime}$. Then $\nu \in \mathfrak{M}\left(\mathfrak{T}_{K}, \rho\right)$.

Proof. We start by recalling the following Theorem of Varadarajan (Theorem 3.2 in [14]).

Theorem 3 (Varadarajan). Assume that a locally compact second countable group $K$ acts measurably on a standard Borel space $(X, \mathcal{B})$. There exists a compact metric space $Z$, a continuous action of $K$ on $Z$ and a $K$ invariant Borel subset $Z^{\prime} \subset Z$ such that the restricted action of $K$ on $Z^{\prime}$ is measurably isomorphic to the action of $K$ on $(X, \mathcal{B})$. 
Question. Under what assumptions does the same conclusion hold for Borel actions of inductively compact groups?

We apply Varadarajan's Theorem to the action of our compact group $K$. Passing, if necessary, to the larger space given by the theorem, we may assume that $X$ is a compact metric space, $\nu$ a Borel probability measure, and that the action of $K$ on $X$ is continuous. Consequently, if $k_{n} \rightarrow k_{\infty}$ in $K$ as $n \rightarrow \infty$, then

$$
\nu \circ T_{k_{n}} \rightarrow \nu \circ T_{k_{\infty}}
$$

weakly in the space of Borel probability measures on $X$. It remains to show that the measures $\nu=\rho\left(k_{n}, x\right) \cdot \nu$ weakly converge to the measure $\rho(k, x) \cdot \nu$ as $n \rightarrow \infty$, and the equality $\nu \circ T_{k_{\infty}}=\rho\left(k_{\infty}, x\right) \cdot \nu$ will be established. First of all, observe that the function

$$
\rho_{\max }(x)=\max _{k \in K} \rho(k, x)
$$

is well-defined and measurable in $X$ (since, by continuity, the maximum can be replaced by the supremum over a countable dense set). We shall show that for any bounded measurable function $\psi$ on $X$ we have

$$
\lim _{n \rightarrow \infty} \int_{X} \psi(x) \rho\left(k_{n}, x\right) d \nu(x)=\int_{X} \psi(x) \rho\left(k_{\infty}, x\right) d \nu(x) .
$$

Assume $\psi$ satisfies $0 \leq \psi \leq 1$. For every $x \in X$ we have

$$
\lim _{n \rightarrow \infty} \rho\left(k_{n}, x\right)=\rho\left(k_{\infty}, x\right) \text {. }
$$

By Fatou's Lemma,

$$
\int \psi(x) \rho\left(k_{\infty}, x\right) d \nu(x) \leq \lim _{n \rightarrow \infty} \inf \int \psi(x) \rho\left(k_{n}, x\right) d \nu(x) .
$$

For $N>0$ set $X_{N}=\left\{x: \rho_{\max }(x) \leq N\right\}$. Take $\varepsilon>0$ and choose $N$ large enough in such a way that we have

$$
\nu\left(X \backslash X_{N}\right)<\varepsilon, \quad \int_{X \backslash X_{N}} \psi(x) \rho\left(k_{\infty}, x\right) d \nu(x)<\varepsilon .
$$

Observe that since $X_{n}$ is $K$-invariant, for all $n \in \mathbb{N}$ we have

$$
\int_{X \backslash X_{N}} \psi(x) \rho\left(k_{n}, x\right) d \nu(x) \leq \nu \circ T_{k_{n}}\left(X \backslash X_{N}\right)=\nu\left(X \backslash X_{N}\right)<\varepsilon .
$$

By the bounded convergence theorem, we have

$$
\lim _{n \rightarrow \infty} \int_{X_{N}} \psi(x) \rho\left(k_{n}, x\right) d \nu(x)=\int_{X_{N}} \psi(x) \rho\left(k_{\infty}, x\right) d \nu(x),
$$


whence

$$
\int_{X} \psi(x) \rho\left(k_{\infty}, x\right) d \nu(x) \geq \lim _{n \rightarrow \infty} \sup \int \psi(x) \rho\left(k_{n}, x\right) d \nu(x)-3 \varepsilon .
$$

Since $\varepsilon$ is arbitrary, the proposition is proved.

We return to the proof of the second claim of Proposition 12 .

First, take $n_{0} \in \mathbb{N}$ and show that for $\widetilde{\nu}$-almost every $\mathcal{C}$ and all $k \in K\left(n_{0}\right)$ we have

$$
\frac{d \nu_{e} \circ T_{k}}{d \nu_{e}}=\rho(k, x)
$$

Choose a countable dense subgroup $K^{\prime} \subset K\left(n_{0}\right)$. The equality (17) holds for all $k \in K^{\prime}$ and for $\widetilde{\nu}$-almost all $\mathrm{C}$. But then fibrewise continuity of the cocycle $\rho$ implies that (17) holds also for all $k \in K\left(n_{0}\right)$. Consequently, $\nu_{\mathrm{C}} \in \mathfrak{M}(\rho, \mathfrak{T})$ for $\widetilde{\nu}$-almost all $\mathcal{C}$. Now, by definition of the partition $\xi$, for every $\varphi \in \Phi$ we have

$$
\mathcal{A}_{\infty}^{\rho}=\int \varphi d \nu_{\mathrm{e}}
$$

almost surely with respect to $\nu_{\mathcal{C}}$ (indeed, the function $\mathcal{A}_{\infty}^{\rho} \varphi$ is almost surely constant in restriction to $\mathcal{C}$, but then the constant must be equal to the average value).

Since $\Phi$ is dense in $L_{1}\left(X, \nu_{\mathcal{e}}\right)$, and $\nu_{\mathcal{e}} \in \mathfrak{M}(\mathfrak{T}, \rho)$, we conclude that $\nu_{\mathrm{e}}$ is ergodic for $\widetilde{\nu}$-almost every $\mathcal{C}$, and the Proposition is proved completely.

\subsection{Uniqueness of the ergodic decomposition. Consider the map}

$$
\text { Mes }: \mathfrak{M}(\mathfrak{T}, \rho) \rightarrow \mathfrak{M}(\mathfrak{M} \text { erg }(\mathfrak{T}, \rho))
$$

that to a measure $\nu \in \mathfrak{M}(\mathfrak{T}, \rho)$ assigns the measure

$$
\bar{\nu}=\operatorname{Mes}(\nu)=\left(\operatorname{Mes}_{\xi^{\nu}}\right)_{*} \nu .
$$

By definition, we have

$$
\nu=\int_{\mathfrak{M}_{\operatorname{erg}}(\mathfrak{T}, \rho)} \eta d \bar{\nu}(\eta) .
$$

Conversely, introduce a map $E D: \mathfrak{M}\left(\mathfrak{M}_{\text {erg }}(\mathfrak{T}, \rho)\right) \rightarrow \mathfrak{M}(\mathfrak{T}, \rho)$ which takes a measure $\bar{\nu} \in \mathfrak{M}\left(\mathfrak{M}_{\text {erg }}(\mathfrak{T}, \rho)\right)$ to the measure $\nu$ given by the formula (18).

We now check that the maps $E D$ and Mes are both Borel measurable and are inverses of each other. It is clear by definition that the map $E D$ is Borel measurable and that $E D \circ$ Mes $=I d$. We proceed to the proof of the remaining claims. 
First we check that the map Mes is Borel measurable. Indeed, take $\alpha_{1}, \alpha_{2} \in \mathbb{R}$, take a set $A \in \mathcal{B}(X)$ and consider the set $\tilde{A}_{\alpha_{1}, \alpha_{2}} \subset \mathfrak{M}\left(\mathfrak{M}_{\mathrm{erg}}(\mathfrak{T}, \rho)\right)$ given by the formula

$\tilde{A}_{\alpha_{1}, \alpha_{2}}=\left\{\bar{\nu} \in \mathfrak{M}\left(\mathfrak{M}_{\operatorname{erg}}(\mathfrak{T}, \rho)\right): \bar{\nu}\left(\left\{\eta \in \mathfrak{M}_{\operatorname{erg}}(\mathfrak{T}, \rho): \eta(A)>\alpha_{1}\right\}\right)>\alpha_{2}\right\}$.

It is clear that

$(\text { Mes })^{-1}\left(\tilde{A}_{\alpha_{1}, \alpha_{2}}\right)=\left\{\nu \in \mathfrak{M}(\mathfrak{T}, \rho): \nu\left(\left\{x \in X: \mathcal{A}_{\infty}^{\rho} \chi_{A}(x)>\alpha_{1}\right\}\right)>\alpha_{2}\right\}$,

and measurability of the map Mes is proved.

It remains to show that for a given measure $\nu \in \mathfrak{M}(\mathfrak{T}, \rho)$ there is only one measure $\bar{\nu} \in \mathfrak{M}\left(\mathfrak{M}_{\operatorname{erg}}(\mathfrak{T}, \rho)\right)$ such that $\nu=E D(\bar{\nu})$ - namely, $\bar{\nu}=$ $\operatorname{Mes}(\nu)$. To prove this invertibility of the map $E D$ it suffices to establish the following

Proposition 16. Let $\bar{\nu}_{1}, \bar{\nu}_{2} \in \mathfrak{M}(\mathfrak{M} \operatorname{erg}(\mathfrak{T}, \rho))$. If $\bar{\nu}_{1} \perp \bar{\nu}_{2}$, then also $E D\left(\bar{\nu}_{1}\right) \perp$ $E D\left(\bar{\nu}_{2}\right)$.

Proof. Let $\nu_{0}=E D\left(\left(\bar{\nu}_{1}+\bar{\nu}_{2}\right) / 2\right)$, and let $A_{1}, A_{2} \subset \mathfrak{M}\left(\mathfrak{M}_{\text {erg }}(\mathfrak{T}, \rho)\right)$ be disjoint sets satisfying

$$
\bar{\nu}_{1}\left(A_{1}\right)=\bar{\nu}_{2}\left(A_{2}\right)=1 ; \bar{\nu}_{1}\left(A_{2}\right)=\bar{\nu}_{2}\left(A_{1}\right)=0 .
$$

The sets $X_{1}=\left(\operatorname{Mes}_{\xi^{\nu_{0}}}\right)^{-1}\left(A_{1}\right), X_{2}=\left(\operatorname{Mes}_{\xi^{\nu_{0}}}\right)^{-1}\left(A_{2}\right)$ are then disjoint and $\nu_{0}$-measurable. Furthermore, by definition we have

$$
E D\left(\bar{\nu}_{1}\right)\left(X_{1}\right)=E D\left(\bar{\nu}_{2}\right)\left(X_{2}\right)=1 ; E D\left(\bar{\nu}_{1}\right)\left(X_{2}\right)=E D\left(\bar{\nu}_{2}\right)\left(X_{1}\right)=0,
$$

whereby the Proposition is proved and the uniqueness of the ergodic decomposition is fully established.

\section{Proof of Theorem 2}

In the proof of Corollary 1 we have constructed an ergodic decomposition

$$
\nu=\int_{\substack{\infty \\ \mathfrak{M}_{f, 1, e r g}}} \eta d \tilde{\nu}(\eta)
$$

where the measure $\tilde{\nu} \in \mathfrak{M}\left(\mathfrak{M}_{f, 1, \text { erg }}^{\infty}\right)$ is automatically admissible.

Given any positive measurable function $\varphi: \mathbb{P}^{\infty} \rightarrow \mathbb{R}_{>0}$, we can deform the decomposition (19) by writing

$$
\nu=\int_{\mathfrak{M}_{f, 1, e r g}^{\infty}} \frac{\eta}{\varphi(\mathbf{p}(\eta))} \varphi(\mathbf{p}(\eta)) d \tilde{\nu}(\eta) .
$$


Conversely, for any $\sigma$-finite measure $\nu^{\prime} \in \mathfrak{M}^{\infty}\left(\mathbb{P} \mathfrak{M}^{\infty}\right)$ satisfying $\left[\nu^{\prime}\right]=$ $\left[\mathbf{p}_{*} \tilde{\nu}\right]$, we can immediately give a measure $\check{\nu} \in \mathfrak{M}^{\infty}\left(\mathfrak{M}^{\infty}\right)$ such that $\mathbf{p}_{*} \check{\nu}=$ $\tilde{\nu}$ and

$$
\nu=\int_{\mathfrak{M} \infty} \eta d \check{\nu}(\eta)
$$

Since $\tilde{\nu}$ is admissible, the measure $\check{\nu}$ with the desired properties is clearly unique.

To complete the proof, we must now show that the measure class $\left[\mathbf{p}_{*} \bar{\nu}\right]$ is the same for all admissible measures $\bar{\nu}$ occurring in the ergodic decomposition of the given measure $\nu$.

Recall that the map $P_{f}: \mathfrak{M}_{f}^{\infty} \longrightarrow \mathfrak{M}$ is defined by the formula

$$
P_{f}(\nu)=\frac{f \nu}{\nu(f)}
$$

For $\lambda \in \mathbb{R}_{+}$we clearly have

$$
P_{f}(\lambda \nu)=P_{f}(\nu)
$$

The map $P_{f}$ therefore induces a map from $\mathbb{P}_{\mathfrak{M}_{f}^{\infty}}^{\infty}$ to $\mathfrak{M}$, for which we keep the same symbol.

The map $P_{f}: \mathbb{P M}_{f}^{\infty} \rightarrow \mathfrak{M}$ is invertible: the inverse is the map that to a measure $\nu \in \mathfrak{M}$ assigns the projective equivalence class of the measure $\frac{\nu}{f}$.

By definition, given any ergodic decomposition

$$
\nu=\int_{\mathfrak{M}^{\infty}} \eta d \tilde{\nu}(\eta)
$$

of a measure $\nu \in \mathfrak{M}_{f}^{\infty}$, for the measure $\tilde{\nu} \in \mathfrak{M}\left(\mathfrak{M}^{\infty}\right)$ we have

$$
\tilde{\nu}\left(\mathfrak{M}_{f, \text { erg }}^{\infty}\right)=1
$$

Take therefore an ergodic decomposition

$$
\nu=\int_{\mathfrak{M}_{\mathrm{erg}, g}^{\infty}} \eta d \tilde{\nu}(\eta)
$$

Applying the map $P_{f}$, write

$$
P_{f} \nu=\int_{\substack{\mathfrak{M}_{\mathrm{erg}, f}^{\infty}\\}} P_{f} \eta \cdot \frac{\eta(f)}{\nu(f)} d \tilde{\nu}(\eta) .
$$

The measure

$$
\frac{\eta(f)}{\nu(f)} d \tilde{\nu}(\eta)
$$

is a probability measure on $\mathfrak{M}_{\text {erg,f }}^{\infty}$ since so is $P_{f} \eta$ for any $\eta \in \mathfrak{M}_{f}^{\infty}$. 
Introduce a measure $\check{\nu} \in \mathfrak{M}\left(\mathfrak{M}_{\mathrm{erg}, f}^{\infty}\right)$ by the formula

$$
d \check{\nu}(\eta)=\frac{\eta(f)}{\nu(f)} d \tilde{\nu}(\eta)
$$

and rewrite (22) as follows:

$$
P_{f} \nu=\int_{\mathfrak{M}} \eta d\left(\left(P_{f}\right)_{*} \check{\nu}\right) .
$$

By definition, the formula (23) yields an ergodic decomposition of the measure $P_{f} \nu \in \mathfrak{M}\left(\mathfrak{T}, \rho_{f}\right)$, indeed, the measure $\left(P_{f}\right)_{*} \check{\nu}$ is by definition supported on $\mathfrak{M}_{\text {erg }}\left(\mathfrak{T}, \rho_{f}\right)$. Since ergodic decomposition is unique in $\mathfrak{M}\left(\mathfrak{T}, \rho_{f}\right)$, we obtain that the measure $\left(P_{f}\right)_{*} \check{\nu}$ does not depend on a specific initial ergodic decomposition (21).

From the clear equality $[\check{\nu}]=[\tilde{\nu}]$ it follows that

$$
\left[\left(P_{f}\right)_{*} \check{\nu}\right]=\left[\left(P_{f}\right)_{*} \tilde{\nu}\right]
$$

and, consequently, the measure class $\left[\left(P_{f}\right)_{*} \tilde{\nu}\right]$ does not depend on the specific choice of an ergodic decomposition (21).

Now recall that the map $P_{f}$ induces a Borel isomorphism between Borel spaces $\mathbb{P M}_{f}^{\infty}$ and $\mathfrak{M}$. Since the measure class $\left[\left(P_{f}\right)_{*} \tilde{\nu}\right]$ does not depend on the specific choice of an ergodic decomposition, the same is also true for the measure class $\left[\mathbf{p}_{*} \tilde{\nu}\right]$. The Proposition is proved completely.

4.1. Finite and infinite ergodic components. Ergodic components of an infinite $G$-invariant measure can be both finite and infinite, and the preceding results immediately imply the following description of the sets on which finite and infinite ergodic componets of an inifnite invariant measure are supported.

Corollary 4. Let $\mathfrak{T}$ be a measurable action of an inductively compact group $G$ on a standard Borel space $(X, \mathcal{B})$, and let $\nu$ be a $\sigma$-finite $\mathfrak{T}$-invariant Borel measure on $X$ such that the space $L_{1}(X, \nu)$ contains a positive Borel measurable fibrewise continuous function. There exist two disjoint Borel $G$-invariant subsets $X_{1}, X_{2}$ of $X$ satisfying $X_{1} \cup X_{2}=X$ and such that the following holds.

(1) There exists a family $Y_{n}$ of Borel G-invariant subsets satisfying $\nu\left(Y_{n}\right)<$ $+\infty$ and such that

$$
X_{1}=\bigcup_{n} Y_{n}
$$

If $Y$ is a Borel $G$-invariant subset satisfying $\nu(Y)<+\infty$, then $\nu\left(X_{1} \backslash Y\right)=0$. With respect to any ergodic decomposition, almost all ergodic components of the measure $\left.\nu\right|_{X_{1}}$ are finite. 
(2) If $\varphi$ is a bounded measurable function, supported on $X_{2}$ and squareintegrable with respect to $\nu$, then for the corresponding sequence of averages we have $\mathcal{A}_{n} \varphi \rightarrow 0$ in $L_{2}(X, \nu)$. With respect to any ergodic decomposition, almost all ergodic components of the measure $\left.\nu\right|_{X_{2}}$ are infinite.

By definition, the sets $X_{1}, X_{2}$ are unique up to subsets of measure zero.

In the case of continuous actions, a following description can also be given. Let $X$ be a complete separable metric space, and let $\nu$ be a Borel measure that assigns finite weight to every ball. Given a point $x \in x$, introduce the orbital measures $\eta_{x}^{n}$ by the formula

$$
\eta_{x}^{n}=\int_{K(n)} \delta_{T_{k} x} d \mu_{K(n)}(k) .
$$

Equivalently, for any bounded continuous function $f$ on $X$, we have

$$
\int_{X} f d \eta_{x}^{n}=\int_{K(n)} f\left(T_{k} x\right) d \mu_{K(n)}(k) .
$$

In this case the sets $X_{1}, X_{2}$ admit the following characterization: the set $X_{1}$ is the set of all $x$ for which the sequence $\eta_{x}^{n}$ weakly converges to a probability measure as $n \rightarrow \infty$, while the set $X_{2}$ is the set of all $x$ such that for any bounded continuous function $f$ on $X$ whose support is a bounded set, we have

$$
\lim _{n \rightarrow \infty} \int_{X} f d \eta_{x}^{n}=0 .
$$

\section{KOLMOGOROV'S EXAMPLE AND PROOF OF PROPOSITION 1 ,}

5.1. Kolmogorov's Example. For completeness of the exposition we briefly recall Kolmogorov's example [5] showing that, for actions of large groups, ergodic invariant probability measures may fail to be indecomposable.

Let $G$ be the group of all bijections of $\mathbb{Z}$, and let $\Omega_{2}$ be the space of bi-infinite binary sequences. The group $G$ acts on $\Omega_{2}$ and preserves any Bernoulli measure on $\Omega_{2}$.

Let $G_{0} \subset G$ be the subgroup of finite permutations, that is, permutations that only move a finite subset of symbols. The group $G_{0}$ is inductively compact. De Finetti's Theorem states that $G_{0}$-invariant indecomposable (or, equivalently, ergodic) probability measures on $\Omega_{2}$ are precisely the Bernoulli measures.

It follows that $G$-invariant indecomposable probability measures are precisely Bernoulli measures as well. Nonetheless, if $\nu_{1}$ and $\nu_{2}$ are two distinct non-atomic Bernoulli measures on $\Omega_{2}$, then the measure $\frac{\nu_{1}+\nu_{2}}{2}$ is ergodic! 
Indeed, the group $G$ has only countably many orbits on $\Omega_{2}$ and it is easily verified that any $G$-invariant set must have either full or zero measure with respect to $\frac{\nu_{1}+\nu_{2}}{2}$.

5.2. Proof of Proposition 1. As before, let $(X, \mathcal{B})$ be a standard Borel space. Let $G$ be an arbitrary group, and let $\mathfrak{T}$ be an action of $G$ on $X$. The action $\mathfrak{T}$ will be called weakly measurable if for any $g \in G$ the transformation $T_{g}$ is Borel measurable. Similarly, a positive multiplicative cocycle

$$
\rho: G \times X \longrightarrow \mathbb{R}_{>0}
$$

will be called weakly measurable if for any $g \in G$ the function $\rho(g, x)$ is Borel measurable in $x$. For a weakly measurable cocycle $\rho$ the space $\mathfrak{M}(\mathfrak{T}, \rho)$ is defined in the same way and is again a convex cone. A measure $\nu \in \mathfrak{M}(\mathfrak{T}, \rho)$ will be called strongly indecomposable if a representation

$$
\nu=\alpha \nu_{1}+(1-\alpha) \nu_{2}
$$

with $\nu_{1}, \nu_{2} \in \mathfrak{M}(\mathfrak{T}, \rho), \alpha \in(0,1)$ is only possible when $\nu=\nu_{1}=\nu_{2}$. A measure $\nu$ will be called weakly indecomposable if for any Borel measurable set $A$ satisfying, for every $g \in G$, the condition $\nu\left(A \triangle T_{g} A\right)=0$, we must have $\nu(A)=0$ or $\nu(A)=1$.

Proposition 17. A measure $\nu \in \mathfrak{M}(\mathfrak{T}, \rho)$ is weakly indecomposable if and only if it is strongly indecomposable.

It is more convenient to prove the following equivalent reformulation.

Proposition 18. Let $\rho$ be a positive multiplicative weakly measurable cocycle over a weakly measurable action of a group $G$ on a standard Borel space $(X, \mathcal{B})$. Let $\nu_{1}, \nu_{2} \in \mathfrak{M}(\mathfrak{T}, \rho)$ be weakly indecomposable. Then either $\nu_{1}=\nu_{2}$ or $\nu_{1} \perp \nu_{2}$.

Proof. Indeed, let $\nu_{1}, \nu_{2} \in \mathfrak{M}(\mathfrak{T}, \rho)$ be weakly indecomposable. Consider the Jordan decomposition of $\nu_{1}$ with respect to $\nu_{2}$ and write

$$
\nu_{1}=\widetilde{\nu}_{2}+\nu_{3}, \quad \widetilde{\nu}_{2} \ll \nu_{2}, \quad \nu_{3} \perp \nu_{2} .
$$

Since $\nu_{2} \circ T_{g} \ll \nu_{2}$, we also have $\nu_{2}\left(\backslash T_{g} A\right)=0$ for each $g \in G$. It follows that for each $g \in G$ we have $\nu_{1}\left(A \triangle T_{g} A\right)=0$, whence either $\nu_{1}(A)=0$ or $\nu_{1}(A)=1$. If $\nu_{1}(A)=0$, then $\nu_{1} \perp \nu_{2}$, and we are done. If $\nu_{1}(A)=1$, then $\nu_{3}=0$, and we have $\nu_{1} \ll \nu_{2}$. Set

$$
\varphi=\frac{d \nu_{1}}{d \nu_{2}}
$$

Since $\nu_{1}, \nu_{2} \in \mathfrak{M}(\mathfrak{T}, \rho)$ and $\nu_{1} \ll \nu_{2}$, for each $g \in G$ the function $\varphi$ satisfies, $\nu_{2}$-almost surely, the equality

$$
\varphi\left(T_{g} x\right)=\varphi(x)
$$


But then, the weak indecomposability of $\nu_{2}$ implies that $\varphi=1$ almost surely with respect to $\nu_{2}$, and, therefore, $\nu_{1}=\nu_{2}$. The Proposition is proved completely.

\section{REFERENCES}

[1] V.I. Bogachev, Measure theory, Springer Verlag, 2007.

[2] Borodin, Alexei; Olshanski, Grigori Infinite random matrices and ergodic measures. Comm. Math. Phys. 223 (2001), no. 1, $87-123$.

[3] A.Bufetov, Finiteness of ergodic unitarily invariant measures on spaces of infinite matrices, arXiv: 1108.2737

[4] Y. Coudène, Systèmes dynamiques et théorie ergodique, notes du cours de DEA.

[5] Fomin, S. V. On measures invariant under certain groups of transformations. (Russian) Izvestiya Akad. Nauk SSSR. Ser. Mat. 14, (1950), 261 - 274.

[6] Greschonig, Gernot; Schmidt, Klaus Ergodic decomposition of quasi-invariant probability measures. Dedicated to the memory of Anzelm Iwanik. Colloq. Math. 84/85 (2000), part 2, $495-514$.

[7] Kifer, Ju. I.; Pirogov, S. A. The decomposition of quasi-invariant measures into ergodic components. (Russian) Uspehi Mat. Nauk 27 (1972), no. 5(167), 239 - 240.

[8] Kryloff, Nicolas; Bogoliouboff, Nicolas, La théorie générale de la mesure dans son application à l'étude des systèmes dynamiques de la mécanique non linéaire, Ann. of Math. (2) 38 (1937), 65 - 113.

[9] Phelps, Robert R. Lectures on Choquet's theorem. Second edition. Lecture Notes in Mathematics, 1757. Springer-Verlag, Berlin, 2001.

[10] Rohlin, V. A. On the fundamental ideas of measure theory. (Russian) Mat. Sbornik N.S. 25(67), (1949), 107 - 150.

[11] Rohlin, V. A. On the decomposition of a dynamical system into transitive components. (Russian) Mat. Sbornik N.S. 25(67), (1949). 235 - 249.

[12] Schmidt, Klaus Cocycles on ergodic transformation groups. Macmillan Lectures in Mathematics, Vol. 1. Macmillan Company of India, Ltd., Delhi, 1977. 202 pp.

[13] Souslin M. Ya., Sur une définition des ensembles mesurables B sans normbres transfinis, C.R. Acad. Sci. Paris 164: 2 (1917), 88-91.

[14] Varadarajan, V. S. Groups of automorphisms of Borel spaces. Trans. Amer. Math. Soc. 1091963191 - 220.

[15] Vershik, A. M. A description of invariant measures for actions of certain infinitedimensional groups. (Russian) Dokl. Akad. Nauk SSSR 218 (1974), 749 - 752.

[16] J. von Neumann, Zur Operatorenmethode in der klassischen Mechanik, Ann. of Math. (2) 33 (1932), $587-642$.

Steklov Institute of Mathematics, Moscow

InSTITUTE FOR INFORMATION TRANSMISSION PROBLEMS, MOSCOW

National Research University Higher School of Economics, Moscow

Rice University, Houston TX 